\title{
Adaptive Sliding Mode Control of a Nonlinear Electro-Hydraulic Servo System for Position Tracking
}

\author{
Mingxing YANG*, Qi ZHANG**, Xinliang LU***, Ruru XI****, Xingsong WANG**** \\ *School of Mechanical Engineering, Southeast University, Nanjing 211189, China, E-mail: mingxingyangvip@163.com \\ **School of Mechanical Engineering, Southeast University, Nanjing 211189, China, E-mail: kichy_zq@163.com \\ ***School of Mechanical Engineering, Shijiazhuang Tiedao University, Shijiazhuang 050043, China, \\ E-mail:xinref@126.com \\ ****School of Mechanical Engineering, Hangzhou Dianzi University, 310018 Hangzhou, China, \\ E-mail:xiruru@hdu.edu.cn \\ ****School of Mechanical Engineering, Southeast University, Nanjing 211189, China, E-mail: xswang@seu.edu.cn \\ cross $^{\text {ref }}$ http://dx.doi.org/10.5755/j01.mech.25.4.22822
}

\section{Introduction}

The electro-hydraulic servo system (EHSS) has been widely employed in the field of engineering due to prominent advantages such as fast dynamic response, small size, large force/torque output and high reliability $[1,2]$. However, it is typically non-linear with various nonlinear nature of hydraulic dynamics due to the nonlinear pressureflow characteristics of servo valve, nonlinear friction and nonlinear dynamics of pressure. Apart from dynamical characteristics of essential nonlinear, the EHSS also has other drawbacks such as parametric uncertainties and external disturbances that caused by surrounding environment [3]. The above-mentioned shortcomings limit the development of high performance controller for conventional valve-controlled hydraulic system. Hence, how to weaken the influences of uncertain nonlinearities and ensure the high tracking accuracy of the EHSS in practical implementations has always been the focus of attention.

Due to their simplicity, the PID controller and the input/output linearizing controller [4-6] are commonly used for position tracking of the EHSS in the situations of low control response and precision. However, linear controllers used in the nonlinear uncertain system may degrade the system performance and robustness [7]. Therefore, in order to obtain higher performance, selecting the appropriate controllers for nonlinear uncertain systems is an important aspect in the field of EHSS. Compared with the traditional linear control methods, non-linear control methods and intelligent approaches demonstrate better sensitivity to both the non-linearity and uncertainty of electro-hydraulic system. Sliding mode control (SMC) [8-10], fuzzy control [11, 12], adaptive control $[4,13-16]$ and neural network control [17, 18] have been successfully utilized in EHSS to meet practical and different control demands, but these methods still have their own limitations. Adaptive control is an effective method to deal with control problems coming from uncertain nonlinear system, especially the system uncertainty caused by uncertain parameters $[19,20]$. However, due to the weak robustness caused by external disturbance, adaptive control is difficult to meet the high stability requirements. With the merits of robustness to parameter variations and insensitivity to disturbances, sliding mode control brings new ideas for nonlinear uncertain systems. Nevertheless, its inherent chattering phenomenon will take place when the system operates near the sliding surface [13]. Although both of the fuzzy control strategy and the neural network can approximate any nonlinear function to a desired accuracy, they may not have enough reliability on account that the former depends seriously on expert experiences and the latter may resulting the "explosion of complexity" problem [21].

From previous findings and discussion, it is reasonable to combine adaptive control techniques and the SMC to treat system parameter uncertainties and external disturbances for nonlinear uncertain systems. Obviously, adaptive sliding mode control (ASMC) is a preferred option with simple control structure, strong adaptability and robustness to parameter variations and external disturbances. The purpose in this paper is to provide a developed adaptive sliding mode controller to realize the high-performance tracking control of EHSS with model uncertainty. In particular, this scheme combines the sliding model control based on modified exponent reaching law with adaptive algorithm. Both the parametric adaptive estimation law and discontinuous projection algorithm are designed to estimate unknown parameters of the subject, which can effectively overcome the influence caused by the parameter uncertainty. The sliding surface with variable gain coefficient is presented for the switching control part of the sliding mode, which combines the idea of saturation function instead of the sign function to eliminate the chattering phenomenon. At last, the comparative experiment is performed to illustrate the control performance of the proposed ASMC strategy.

In general, this paper is organized as follows. Section 2 gives the detailed dynamic models and problem formulation of the EHSS. The controller combines the sliding model control based on modified exponent reaching law with adaptive algorithm is designed and the related stability analysis is given in Section 3. Then the comparative experimental results are presented in Section 4. Finally, the conclusion is drawn in Section 5.

\section{Dynamic models of hydraulic servo system}

As depicted in Fig. 1, the electro-hydraulic servo drive cylinder mainly consists of a single-rod cylinder, a three-position four-way servo valve, and an end-effector with external disturbances. Parameters in the system can be defined as follows: $A_{1}$ and $A_{2}$ are the effective working area at both sides of the piston, $p_{1}$ and $p_{2}$ denote the pressures in 
the cylinder forward and return chamber, respectively, $q_{1}$ and $q_{2}$ are the liquid flow of the two actuator chambers, respectively. Moreover, $m$ and $x_{p}$ represent the mass and displacement of the end-effector, respectively.

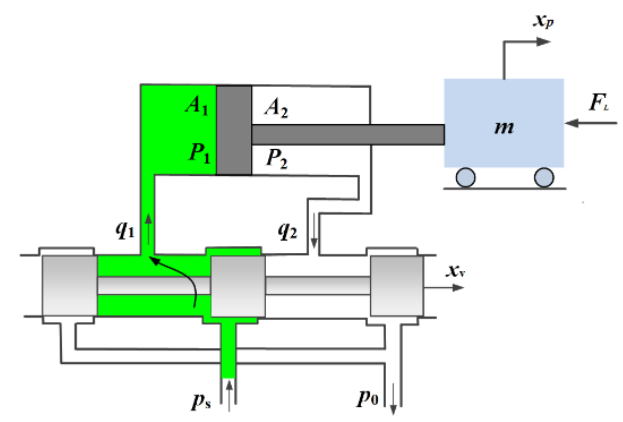

Fig. 1 Configurations of the electro-hydraulic servo drive cylinder

In order to make the end-effector as closely as possible to track the specified motion trajectory, a mathematical model that represents the hydraulic system dynamics is presented as follows. given by:

The dynamics equation of the force balance can be

$$
A_{1} p_{1}-A_{2} p_{2}=F_{L}+k x_{p}+B_{t} \dot{x}_{p}+m \ddot{x}_{p},
$$

where: $F_{L}$ is the external disturbance force, $k$ denotes elastic damping in cylinder and $B_{t}$ represents the viscous damping coefficient caused by hysteretic force-velocity characteristics of the shock absorber.

$$
\text { Define function as } \operatorname{sg}(*)=\left\{\begin{array}{ll}
1, & \text { if } *>0 \\
-1, & \text { if } *<0
\end{array} \text {, and } q_{1}\right.
$$

and $q_{2}$ can be modeled by:

$$
\left\{\begin{array}{l}
q_{1}=C_{d} x_{v} w \sqrt{\left[p_{s}+\operatorname{sg}\left(x_{v}\right)\left(p_{s}-2 p_{1}\right)\right] / \rho} \\
q_{2}=C_{d} x_{v} w \sqrt{\left[p_{s}+\operatorname{sg}\left(x_{v}\right)\left(2 p_{2}-p_{s}\right)\right] / \rho},
\end{array}\right.
$$

where: $C_{d}$ is the discharge coefficient, $x_{v}$ is the servo valve spool displacement, $w$ is the spool valve area gradient and $\rho$ is the fluid density.

In fact, the natural frequency of the servo valve in the experiment is much higher than that of the hydraulic cylinder. On the premise of not significantly reduce the model accuracy, the dynamics of the servo valve can be simplicity approximation by [16]:

$$
x_{v}=K_{v} u
$$

where: $K_{v}$ is a positive constant and $u$ is the control input voltage. Hence, Eq. (2) can be transformed to:

$$
\left\{\begin{array}{l}
q_{1}=C_{d} K_{v} u w \sqrt{\left[p_{s}+\operatorname{sg}(u)\left(p_{s}-2 p_{1}\right)\right] / \rho} \\
q_{2}=C_{d} K_{v} u w \sqrt{\left[p_{s}+\operatorname{sg}(u)\left(2 p_{2}-p_{s}\right)\right] / \rho}
\end{array} .\right.
$$

Neglecting the influence of the fluid temperature and the bulk modulus of elasticity in two chambers, the dynamic equilibrium equations of influent and effluent flow can be expressed as:

$$
\left\{\begin{array}{l}
q_{1}-C_{i}\left(p_{1}-p_{2}\right)-C_{e} p_{1}=A_{1} \dot{x}_{p}+\frac{\left(V_{10}+A_{1} x_{p}\right) \dot{p}_{1}}{\beta_{e}} \\
C_{i}\left(p_{1}-p_{2}\right)-C_{e} p_{2}-q_{2}=A_{2} \dot{x}_{p}+\frac{\left(V_{20}-A_{2} x_{p}\right) \dot{p}_{2}}{\beta_{e}}
\end{array},\right.
$$

where: $V_{i 0}(i=1,2)$ is the initial control volume of the fluid between the valve and the piston; $\beta_{e}$ is the bulk modulus of the fluid; $C_{i}$ and $C_{e}$ are the coefficients of the internal leakage and the external leakage of the cylinder, respectively.

Since the leakage flow is as insignificant as the fluid compression caused by $\beta_{e}$, from Eq. (5) we use the following approximation:

$$
q_{2} / q_{1} \approx A_{2} / A_{1}=\varepsilon
$$

where: $\varepsilon$ denotes the piston area ratio.

In addition, the load pressure $p_{L}$ and the load flow $q_{L}$ of the system are defined as:

$$
\left\{\begin{array}{l}
p_{L}=p_{1}-\varepsilon p_{2} \\
q_{L}=\frac{q_{1}+\varepsilon q_{2}}{1+\varepsilon^{2}}=q_{1}
\end{array} .\right.
$$

When the piston moves slightly at the origin of the displacement, the displacement of the end-effector is close to zero. Taking the Eqs. (5-7) into account, the load pressure $q_{L}$ is rewritten as:

$$
q_{L}=A_{1} \dot{x}_{p}+\lambda_{1} \dot{p}_{L}+\lambda_{2} p_{L}+\lambda_{3} p_{s}
$$

where:

$$
\left\{\begin{array}{l}
\lambda_{1}=\frac{V_{10} V_{20}}{\beta_{e}\left(\varepsilon^{2} V_{10}+V_{20}\right)} \\
\lambda_{2}=\frac{\left(\varepsilon^{3} V_{10}+V_{20}\right)\left(C_{i}+C_{e}\right)+\varepsilon\left(V_{10}+\varepsilon V_{20}\right) C_{i}}{\left(1+\varepsilon^{3}\right)\left(\varepsilon^{2} V_{10}+V_{20}\right)} \\
\lambda_{3}=\frac{\varepsilon\left(V_{20}-V_{10}\right)\left(C_{i}+C_{e}\right)-\left(V_{20}-\varepsilon^{2} V_{10}\right) C_{i}}{\left(1+\varepsilon^{3}\right)\left(\varepsilon^{2} V_{10}+V_{20}\right)} \varepsilon^{1+\operatorname{sg}(u)}
\end{array} .\right.
$$

Taking the starting point of piston rod as its coordinate origin, and from Eq. (2) and Eq. (3), the load pressure $q_{L}$ can be linearized as:

$$
q_{L}=\frac{\partial q_{L}}{\partial x_{v}} x_{v}+\frac{\partial q_{L}}{\partial p_{L}} p_{L}=K_{q} K_{v} u-K_{\mathrm{c}} p_{L}
$$

where: $K_{q}$ and $K_{c}$ are the flow gain coefficient and flowpressure coefficient of the servo valve, respectively. Eq. (10) can be described by:

$$
\left\{\begin{array}{l}
K_{q}=C_{d} w \sqrt{\frac{(1+\varepsilon) p_{s}+\operatorname{sg}(u)\left((1-\varepsilon) p_{s}-2 p_{L}\right)}{\rho\left(1+\varepsilon^{3}\right)}}, \\
K_{c}=\frac{C_{d} K_{v} w u}{\sqrt{\rho\left(1+\varepsilon^{3}\right)\left((1+\varepsilon) p_{s}+\operatorname{sg}(u)\left((1-\varepsilon) p_{s}-2 p_{L}\right)\right)}} .
\end{array}\right.
$$


Finally, define the system state variables as $\left[x_{1}, x_{2}\right.$, $\left.x_{3}\right]^{T}=\left[x_{p}, \dot{x}_{p}, \ddot{x}_{p}\right]^{T}$. Combing Eq. (1) with Eqs. (6) and (7), a mathematical model of the entire system can be formulated by:

$$
\left\{\begin{array}{l}
\dot{x}_{1}=x_{2} \\
\dot{x}_{2}=x_{3} \\
\dot{x}_{3}=\left(-\varphi_{1} x_{1}-\varphi_{2} x_{2}-\varphi_{3} x_{3}+u-d\right) / \beta
\end{array},\right.
$$

where: $u$ is the input of the system, and:

$$
\left\{\begin{array}{l}
\varphi_{1}=\frac{k\left(\lambda_{2}+K_{c}\right)}{A_{1} K_{q} K_{v}} \\
\varphi_{2}=\frac{A_{1}^{2}+B_{t}\left(\lambda_{2}+K_{c}\right)+k \lambda_{1}}{A_{1} K_{q} K_{v}} \\
\varphi_{3}=\frac{\lambda_{1} B_{t}+\left(\lambda_{2}+K_{c}\right) m}{A_{1} K_{q} K_{v}} \\
\beta=\frac{\lambda_{1} m}{A_{1} K_{q} K_{v}} \\
d=\frac{\lambda_{3}}{K_{q} K_{v}} p_{s}+\frac{\left(\lambda_{2}+K_{c}\right) F_{L}+\lambda_{1} \dot{F}_{L}}{A_{1} K_{q} K_{v}}
\end{array}\right.
$$

From Eqs. (11) and (12), it is shown that the hydraulic system can be described as a linear system. However, some internal parameters $C_{i}, C e, \beta_{e}$ and $C_{d}$ are uncertain, due to they are difficult to accurate identify and vary with oil temperature and pressure. Besides, the variety of working conditions and different of forces always cause the external disturbance $d$ cannot be precisely acquired. In respect of the dynamic system shown in the above equations, the following assumption will be made.

Assumption 1. The coefficient $\beta$ is a positive value, and the desired trajectory $x_{d}$, and its first, second and thirdorder derivatives with respect to time are all bounded. In addition, the external disturbance $d$ is limited by $|d| \in \mathrm{D}$. $p_{1}$ and $p_{2}$ are bounded by $p_{\mathrm{s}}$ and $p_{0}$, where $0=p_{0} \leq p_{1}, p_{2} \leq p_{\mathrm{s}}$.

\section{Adaptive sliding model controller design}

\subsection{Sliding mode controller}

Define the tracking error as $e=x_{p}-x_{d}$, and for convenience of writing, $e, \dot{e}$ and $\ddot{e}$ are generally substituted by $e_{1}, e_{2}$ and $e_{3}$, respectively. Thus, in the error state space, a sliding surface $s$ can be chosen as:

$$
s(t)=c_{1} e+c_{2} \dot{e}+\ddot{e}=c_{1} e_{1}+c_{2} e_{2}+e_{3},
$$

where: $c_{1}$ and $c_{2}$ are sliding surface parameters that can be calculated by using the pole collocation method and depended on the desired control performance. Moreover, both of $c_{1}$ and $c_{2}$ are positive constants, and they determine the dynamic performance of the system when the state of the system is located on the sliding surface.

Combine the Eqs. (11) to (13), the time derivative of $s$ can be obtained by: $\dot{s}(t)=c_{1} e_{2}+c_{2} e_{3}+\frac{1}{\beta}\left(-\varphi_{1} x_{1}-\varphi_{2} x_{2}-\varphi_{3} x_{3}+u-d\right)-\dddot{x}_{d}$

In view of the inherent chattering problem in conventional sliding mode control, an exponential audience law is designed for the switch control part. In this way, the time derivative of $s$ is present as:

$$
\dot{s}(t)=-k_{s} s-\eta \operatorname{sgn}(s)
$$

where: $k_{s}$ and $\eta$ are the gain coefficients, and both of them are positive constants.

Furthermore, the switch gain $\eta$ of sliding model control is given a moderate condition by:

$$
\eta-D \geq 0
$$

where: Eq. (16) ensures the system can suppress modeling uncertainty and external disturbance, which have special significance to the performance of the controller. At this point, by combining the sliding model control based on exponent reaching law with adaptive algorithm, we can get:

$$
u=u_{e q}+u_{s m c},
$$

where: $u_{e q}$ and $u_{s m c}$ represent equivalent control term and the switching term of system input, respectively. In particular, the equivalent control term determines the dynamics of the system on the sliding surface since it carries the system state vector over the reference trajectory. And they are defined by:

$$
\left\{\begin{array}{l}
u_{e q}=\varphi_{1} x_{1}+\varphi_{2} x_{2}+\varphi_{3} x_{3}+\beta\left(\dddot{x}_{d}-c_{1} e_{2}-c_{2} e_{3}\right) \\
u_{s m c}=-k_{s} s-\eta \operatorname{sgn}(s)
\end{array} .\right.
$$

A Lyapunov stability theory is constructed to confirm the system stability with the SMC scheme.

Proof 1. Define a Lyapunov candidate function as:

$$
V_{1}=\frac{1}{2} \beta s^{T} s
$$

Inserting Eqs. (16) and (18) into Eq. (19), and differentiating $V_{1}$ with respect to $t$. We obtain:

$$
\begin{aligned}
& \dot{V}_{1}=\beta s\left(c_{1} e_{2}+c_{2} e_{3}-\dddot{x}_{d}\right)-s\left(\varphi_{1} x_{1}+\varphi_{2} x_{2}+\varphi_{3} x_{3}-u+d\right) \leq- \\
& \leq-k_{s} s^{2}-(\eta-D)|s| \leq-k_{s} s^{2} .
\end{aligned}
$$

Hence, according to equations (19) and (20), we can get that $V_{1}$ is positive definite and $\dot{V}_{1}$ is negative definite. Therefore, tracking errors of the end-effecter can converge asymptotically to an adequately small value as the time tends to infinity according to the established SMC law.

\subsection{Parameter adaptive estimation algorithm}

For a practical system of EHSS, the following assumption is first made in this section. 
Assumption 2. The unknown term $d$ is determined by other uncertain parameters and the external disturbance $d_{L}$, and assuming that $d_{\min }$ and $d_{\max }$ are the known lower and upper bounds of $d$. So the bounded $d$ can be designed within a known limit as $d_{\min } \leq d \leq d_{\max }$. To simplify this discussion, an appropriate positive constant $D$ is given to satisfy $-D \leq d_{\min }$ and $d_{\max } \leq D$. At this point, we can get $|d| \leq D$.

In the real implementations of the proposed control strategy in this paper, the SMC controller is expanded with an improved adaptive algorithm to estimate the unknown parameters of the system. Thus, assuming that the uncertainty values $\beta$ and $\varphi_{i}(i=1,2,3)$ are estimated by $\hat{\beta}$ and $\hat{\varphi}_{i}$, so:

$$
\left\{\begin{array}{l}
\tilde{\varphi}_{i}=\varphi_{i}-\hat{\varphi}_{i} \\
\tilde{\beta}=\beta-\hat{\beta}
\end{array}\right.
$$

where: $\tilde{\beta}$ and $\tilde{\varphi}_{i}$ are the ideal estimation errors of $\beta$ and $\varphi_{i}$. Furthermore, choosing that the adjustable parameters can be automatically updated by the following adaptation laws as:

$$
\left\{\begin{array}{l}
\dot{\hat{\varphi}}_{1}=-\gamma_{1} s x_{1} \\
\dot{\hat{\varphi}}_{2}=-\gamma_{2} s x_{2} \\
\dot{\hat{\varphi}}_{3}=-\gamma_{3} s x_{3} \\
\dot{\hat{\beta}}=-\gamma_{4} s\left(\dddot{x}_{d}-c_{1} e_{2}-c_{2} e_{3}\right)
\end{array},\right.
$$

where: $\gamma_{i}(i=1,2,3,4)$ represent the strict positive constants related to the adaptation law. Then, combining Eq. (18) with Eq. (21), the expression for the input of the adaptive sliding controller can be shown as:

$$
\begin{aligned}
& \hat{u}=\hat{u}_{e q}+u_{s m c}= \\
& =\hat{\varphi}_{1} x_{1}+\hat{\varphi}_{2} x_{2}+\hat{\varphi}_{3} x_{3}+\hat{\beta}\left(\dddot{x}_{d}-c_{1} e_{2}-c_{2} e_{3}\right)-k_{s} s-\eta \operatorname{sgn}(s),
\end{aligned}
$$

Finally, a Lyapunov stability theory is constructed to confirm the system stability with the ASMC scheme.

Proof 2. Let, the Lyapunov candidate function chosen as:

$$
V_{2}=\frac{1}{2} \beta s^{2}+\sum_{i=1}^{3} \frac{1}{2 \gamma_{i}} \tilde{\varphi}_{i}^{2}+\frac{1}{2 \gamma_{4}} \tilde{\beta}^{2}
$$

is:

Then, the derivative of the $V_{2}$ with respect to time

$$
\begin{aligned}
& \dot{V}_{2}=\beta s \dot{s}-\sum_{i=1}^{3} \frac{1}{\gamma_{i}} \tilde{\varphi}_{i} \dot{\hat{\varphi}}_{i}-\frac{1}{\gamma_{4}} \tilde{\beta} \dot{\hat{\beta}}=\beta s\left[c_{1} e_{2}+c_{2} e_{3}-\dddot{x}_{d}-\beta^{-1}\left(\sum_{i=1}^{3} \varphi_{i} x_{i}-u+d\right)\right]-\sum_{i=1}^{3} \frac{1}{\gamma_{i}} \tilde{\varphi}_{i} \dot{\hat{\varphi}}_{i}-\frac{1}{\gamma_{4}} \tilde{\beta} \dot{\hat{\beta}}= \\
& =s\left[(\beta-\hat{\beta})\left(c_{1} e_{2}+c_{2} e_{3}-\dddot{x}_{d}\right)-\sum_{i=1}^{3}\left(\varphi_{i}-\hat{\varphi}_{i}\right) x_{i}-k_{s} s-\eta \operatorname{sgn}(s)-d\right]-\sum_{i=1}^{3} \frac{1}{\gamma_{i}} \tilde{\varphi}_{i} \dot{\hat{\varphi}}_{i}-\frac{1}{\gamma_{4}} \tilde{\beta} \dot{\hat{\beta}}= \\
& =s\left[\tilde{\beta}\left(c_{1} e_{2}+c_{2} e_{3}-\dddot{x}_{d}\right)-\sum_{i=1}^{3} \tilde{\varphi}_{i} x_{i}-k_{s} s-\eta \operatorname{sgn}(s)-d\right]-\sum_{i=1}^{3} \frac{1}{\gamma_{i}} \tilde{\varphi}_{i} \dot{\hat{\varphi}}_{i}-\frac{1}{\gamma_{4}} \tilde{\beta} \hat{\hat{\beta}}=\tilde{\beta}\left[\left(c_{1} e_{2}+c_{2} e_{3}-\dddot{x}_{d}\right) s-\right. \\
& \left.-\frac{1}{\gamma_{4}} \dot{\hat{\beta}}\right]-k_{s} s^{2}-\eta|s|-d s-\sum_{i=1}^{3} \tilde{\varphi}_{i}\left(s x_{i}+\frac{1}{\gamma_{i}} \dot{\hat{\varphi}}_{i}\right) .
\end{aligned}
$$

When Eq. (22) is introduced into Eq. (25), Eq. (25) will be transformed into:

$$
\dot{V}_{2}=-k_{s} s^{2}-\eta|s|-d s \leq-k_{s} s^{2}-(\eta-D)|s| \leq-k_{s} s^{2} \leq 0,
$$

which implies $\dot{V}_{2}=0$ as long as $s=0$, ie. when $\dot{V}=0$ and then $s=0$. According to LaSalle invariance principle, the closed-loop system is asymptotically stable that $s \rightarrow 0$ as $t \rightarrow \infty$. Thus, from the adaptive law in Eq. (22) and the control input in Eq. (23), it is concluded that the EHSS will converge to zero along the sliding mode switching plane in a limited time.

Remark 1. In spite of the demonstrated properties of the controller, the system may exhibits obvious chattering phenomenon due to the inaccurate model and large disturbance. Based on this, $\hat{\beta}$ and $\hat{\varphi}_{i}(i=1,2,3)$ are inevitably affected by tracking error and its corresponding derivatives in the actual physical system of EHSS. Therefore, this problem will overestimate these parameters and result in large oscillation or instability of the system.

In order to avoid excessive estimation of parameters from seriously affecting the stability of controller or even destroying the system, the widely used projection algorithm [14] can be adopted, and the learning law (6) should be modified to:

$\dot{\hat{\varphi}}_{i}=\operatorname{Proj}\left(\gamma_{i} \chi_{i}\right)=\left\{\begin{array}{l}0, \text { if } \hat{\varphi}_{i}=\hat{\varphi}_{i \max } \text { and } \gamma_{i} \chi_{i}>0 \\ 0, \text { if } \hat{\varphi}_{i}=\hat{\varphi}_{i \min } \text { and } \gamma_{i} \chi_{i}<0, \\ \gamma_{i} \chi_{i}, \text { otherwise }\end{array}\right.$

where: $\dot{\hat{\beta}}$ is replaced by $\dot{\hat{\varphi}}_{4}$ easy to express, $\chi_{1}=-s x_{1}$, $\chi_{2}=-s x_{2}, \chi_{3}=-s x_{3}$ and $\chi_{4}=-s\left(\dddot{x}_{d}-c_{1} e_{1}-c_{2} e_{2}\right)$. Moreover, the inequality $\varphi_{\text {imin }}<\hat{\varphi}_{i}(t)<\varphi_{\text {imax }}$ is established when the initial values of $\hat{\varphi}_{i}$ are described as 
$\varphi_{\text {imin }}<\hat{\varphi}_{i}(0)<\varphi_{\text {imax }}$. Therefore, the projection algorithm (24) can guarantee the boundedness of unknown parameter estimates.

Remark 2. According to Eq. (15), the gain coefficient $k_{s}$ should be large enough to make the system error quick approach the sliding surface. In addition, $k_{s}$ as the slope of sliding mode surface ought to be as small as possible to help gain coefficient $\eta$ system states stay on the sliding surface all the time. Hence, we should adjust them reasonably to prevent unexpected chattering problem to the system. Though the method, adjusting $k_{s}$ by using the fuzzy logic control strategy while replacing the sign function with a saturation function, can somewhat decrease the undesirable chattering problem, it may have restricted due to its disadvantages of computation spending and seriously depend on expert experiences.

Therefore, for the sake of weakening the chattering phenomenon and shortening the time for tracking errors to reach the sliding surface, this paper proposes the idea of adjusting the gain coefficient $k_{s}$ while replacing the sign function with the saturation function. In particular, the switching term of system input $u_{s m c}$ can be given as:

$$
u_{s m c}=\left\{\begin{array}{cc}
\eta \operatorname{sgn}(s)+k_{s} s, & |s|>\sigma \\
\eta \frac{s}{\sigma} & ,|s| \leq \sigma
\end{array},\right.
$$

where: $\sigma$ is a positive constant representing the boundary layer thickness of the saturation function. In Eq. (25), the gain coefficients $\eta$ and $k_{s}$ will be forced award the sliding mode surface at once the error states of the system are away from the surface, and the gain coefficient $\eta$ will keep the system near the sliding surface when the error states of the system are in the neighborhood of the switching surface.

Furthermore, by combining Eqs. (23) and (25), the modified ASMC law can be shown as:

$$
\hat{u}=\left\{\begin{array}{c}
\hat{u}_{e q}-k_{s}-\eta \operatorname{sgn}(s),|s|>\sigma \\
\hat{u}_{e q}-\eta \frac{s}{\sigma},|s| \leq \sigma
\end{array} .\right.
$$
pressed as:

According to Eq. (29), the Eq. (26) will be ex-

$$
\dot{V}_{2}=\left\{\begin{array}{cc}
-k_{s} s^{2}-(\eta+d)|s|, & |s|>\sigma \\
-\eta \frac{s^{2}}{\sigma}-d s & ,|s| \leq \sigma
\end{array} .\right.
$$

Here, according to Eq. (26) that $\dot{V}_{2}=\leq 0$ as long as $|s|>\sigma$, and otherwise $\dot{V}_{2}$ will be $-\eta s^{2} / \sigma-d s$.

Moreover, considering that $|s| \leq \sigma,|d| \leq D$ and $|s| \leq \sigma, \dot{V}_{2} \leq-|s|(\eta-D) \leq 0$. Thus, the Eq. (29) can realize the tracking control that the tracking error system firstly arrive at the sliding mode in the finite time and then reach the origin in the finite time.

\section{Experiments and results}

\subsection{Introduction of experimental platform}

As shown in Fig. 2, the experimental platform is composed of a single-rod hydraulic cylinder, two pressure sensors are used to measure two cylinder chambers, a displacement sensor (KEYENCE IL-300) providing accurate and continuous motion measurement for the end-effector, an electro-hydraulic servo valve ( AVIC FF101-6) utilized in actuation of the hydraulic actuator, the external load is a tension spring which connects respectively with the fixed block and the end-effector, and a hydraulic supply with $P_{s}=4 \mathrm{MPa}$, etc. In order to ensure the control algorithms are conducted in real-time, they are run in Matlab/Simulink Real-time Workshop environment.

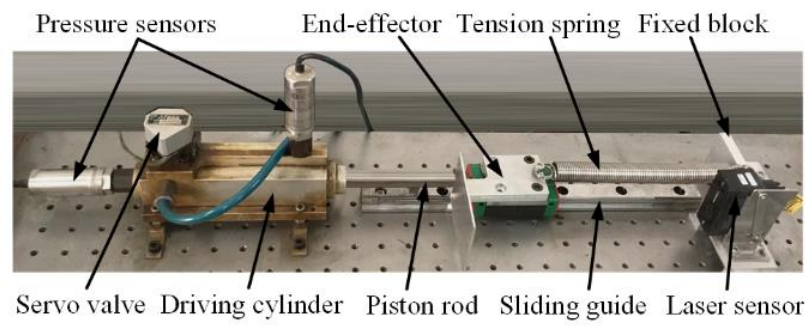

Fig. 2 Experiment platform of the EHSS

Furthermore, a host computer and a target computer are equipped in the industrial PC control system, and they exchange data through serial communication. In this configuration, the analog feedback signal from the displacement sensor is acquired via an analog-to-digital (A/D) card (Advantech PCL-818HD, which can provide 16 channels of single-ended analog input based on ISA bus). After that the acquired analog signals is processed by the designed controller, and the control command $u$ is sent to the digital-toanalog (D/A) card (Advantech PCL-726, with 6 channels and 12 bits analog output channel). Finally, the control output signal is transformed into an analog signal and send to the corresponding servo valve through a power amplifier. The sampling rates for the host and target control layers are all set to $1 \mathrm{kHz}$.

\subsection{Trajectory tracking experiments and results}

Ignoring the influence of damping and the elastic damping in cylinder, details about EHSS parameters are set as follows: $m=4 \mathrm{~kg}, k=0, A_{1}=4.9 \times 10^{-4} \mathrm{~m}^{2}, A_{2}=2.9 \times 10^{-4} \mathrm{~m}^{2}$, $P_{\mathrm{s}}=4 \times 10^{6} \mathrm{~Pa}, \beta_{\mathrm{e}}=8 \times 10^{8} \mathrm{~Pa}, C_{d}=0.8, w=0.012 \mathrm{~m}, C_{i}=5 \times 10^{-}$ ${ }^{13} \mathrm{~m}^{3} /(\mathrm{s} \cdot \mathrm{Pa}), V_{10}=V_{20}=4.5 \times 10^{-5} \mathrm{~m}^{2}, K_{v}=1 \times 10^{-3} \mathrm{~m} / \mathrm{V}$. For the proposed ASMC algorithm, parameters of adaptive laws are set to $\gamma_{1}=0, \gamma_{2}=2 \times 10^{-6}, \gamma_{3}=5 \times 10^{-7}$, and $\gamma_{4}=1 \times 10^{-11}$. The initial and extreme boundary values of system parameters are selected according to Table 1.

In order to illustrate its displacement tracking responses problem, the desired position was guided by a sinusoid wave trajectory as $y=35+25 \sin (\pi t / 2)$. When the system is stable without the external load, the position tracking response and corresponding parameters estimation of EHSS are as shown in Figs. 3 and 4, respectively. As Fig. 3 demonstrates that the cylinder rod can perform well in tracking the desired curve with errors within $1 \mathrm{~mm}$. Meanwhile, the uncertain parameters are dynamic adjusted in certain 
bounded values, as shown in Fig. 4.

Uncertain parameters of the EHSS

\begin{tabular}{cccc}
\hline Parameters & Initial value & Lower value & Upper value \\
\hline$\varphi_{1}$ & 0 & - & - \\
$\varphi_{2}$ & 1025 & 500 & 1200 \\
$\varphi_{3}$ & 125 & 100 & 200 \\
$\varphi_{4}$ & 0.01 & 0.001 & 0.1 \\
\hline
\end{tabular}
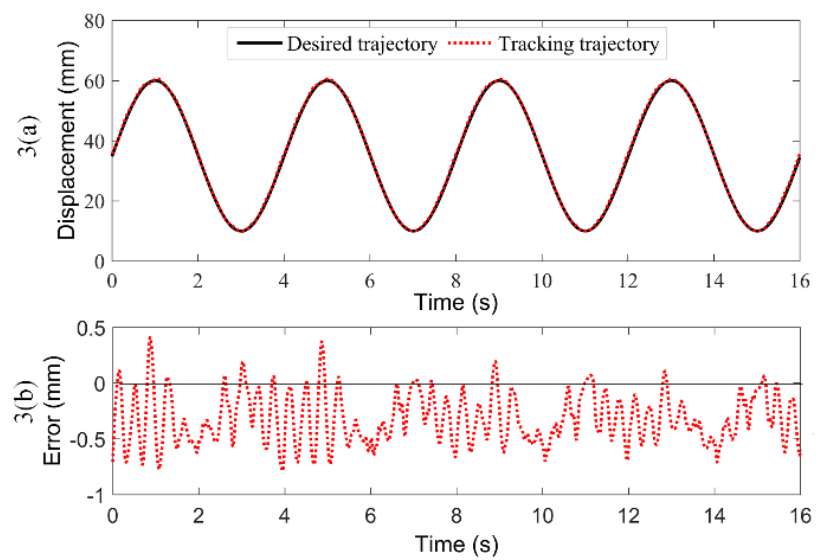

Fig. 3 Position tracking and errors of the developed ASMC controller
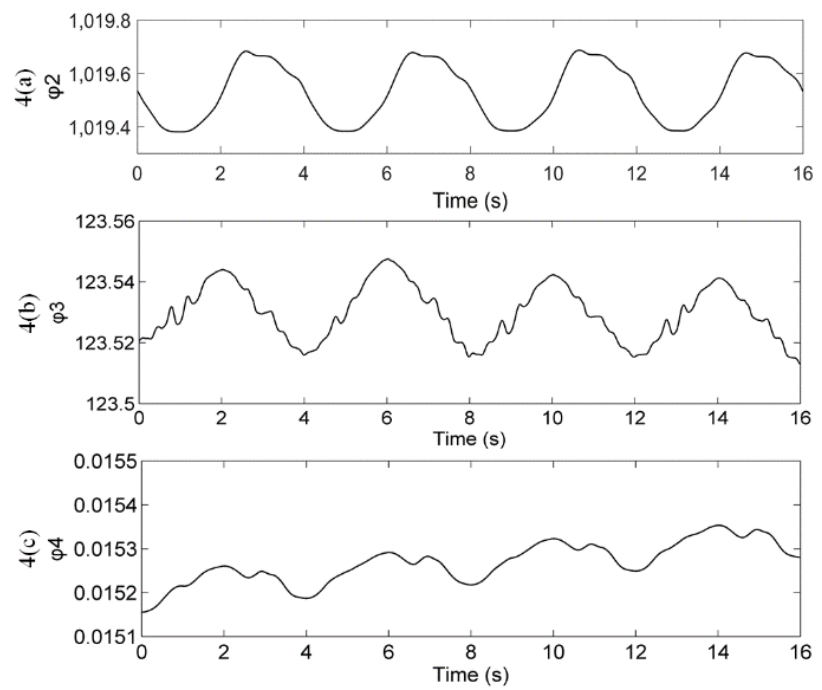

Fig. 4 Estimation values of three uncertain parameters

To validate the effectiveness of the proposed ASMC strategy, a traditional PID controller and an exponent approaching SMC algorithm are conducted to compare with it. In particular, parameters of these two controllers are well-tuned for the sake of best tracking performance. When the elastic load is zero, the motion trajectories and tracking errors of three controllers are shown in Fig. 5. As shown in Fig. 5, a, these controllers can make the end effector of the hydraulic cylinder follow the designed trajectory strictly. The tracking errors of the piston rod is not more than $3 \mathrm{~mm}$ as described in Fig.5b, which indicates that these controllers can obtain good dynamic response performance under no load. Specifically, for PID and SMC, the numerical range of trajectory error is between $-2.6 \mathrm{~mm}$ and $2.2 \mathrm{~mm}$. The tracking error of ASMC is relatively very small, mostly within the measurement resolution of $1 \mathrm{~mm}$, which verifies the high-performance nature of the proposed ASMC control strategy.
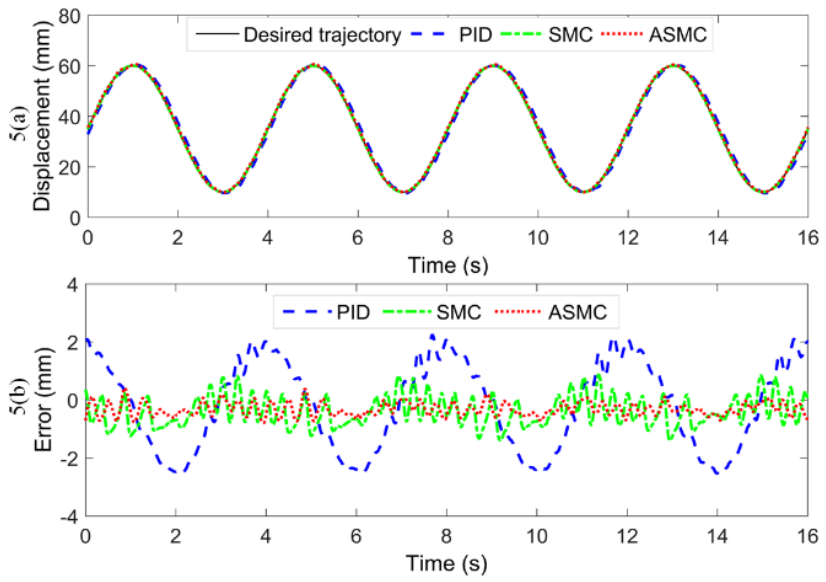

Fig. 5 Comparative results for position tracking control without external disturbance
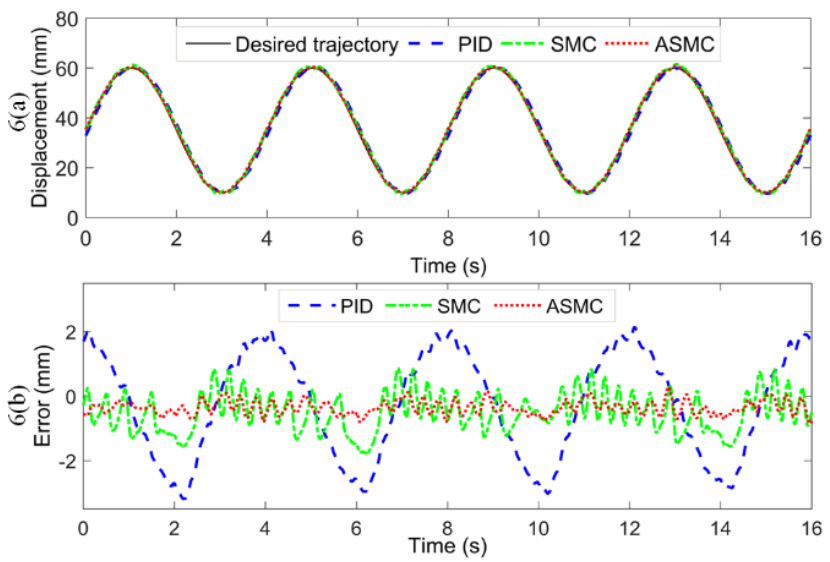

Fig. 6 Comparative results for position tracking control with external disturbance

Furthermore, to evaluate the robustness of the designed controller, the external disturbance is add by a tension spring to the control system. The dynamic behavior of the proposed controller is remaining better than the other two controllers as shown in Fig. 6. As is illustrated in Fig. $6 \mathrm{a}$ and Fig. 6, b, the PID controller cannot handle such a disturbance well and a large tracking error over $3 \mathrm{~mm}$ is exhibited. Though there are also some changes for SMC controller in terms of tracking error, it does not change so much as the PID controller. In contrast, the tracking error of the proposed ASMC is almost invariable. This experimental phenomenon denotes that both of the SMC controller and the proposed ASMC have more capability to suppress disturbance. Moreover, since the parametric estimation laws is used to adapt the actual hydraulic parameter with uncertainties, the proposed controller is more effective for the parametric uncertainties adaptation and load disturbance suppression comparison with the other two controllers.

\section{Conclusion}

In case of external disturbance and model uncertainty, this paper presents a discontinuous projection-based ASMC controller with variable sliding surface gain for position tracking control of an electro-hydraulic servo system. The dynamic model of the valve-controlled system is first established and the corresponding state-space equation is obtained. Next, the parametric adaptive estimation law and discontinuous projection algorithm are designed to estimate 
unknown parameters of the EHSS, which can effectively overcome the influence caused by the parameter uncertainty. After that, the sliding surface with variable gain and the saturation function are designed to modify the controller, with the purpose of solving the chattering problem and improving the robustness of the system. Finally, experimental results demonstrated that the designed controller has better tracking performance when compared with PID controller and the SMC controller.

\section{Acknowledgements}

The authors would like to the China Nation Nature Science Foundation under grant 51575100 and 51805128. Also, all the volunteers who participated in the research are greatly appreciated.

\section{References}

1. Dasmahapatra, S.; Sarkar, B.; Saha, R.; Chatterjee, A. 2015. Design of an adaptive fuzzy-bias SMC and validation for a rugged electrohydraulic system, IEEE/ASME Transactions on Mechatronics 20(6): 2708-2715.

https://doi.org/ 10.1109/TMECH.2015.2393437.

2. Shen, G.; Zhu, Z.; Zhao, J.; Zhu, W. 2016. Real-time tracking control of electro-hydraulic force servo systems using offline feedback control and adaptive control, Isa Transactions 67: 356-370.

https://doi.org/10.1016/j.isatra.2016.11.012.

3. Guo, K.; Wei, J.; Fang, J.; Wang, X. 2015. Position tracking control of electro-hydraulic single-rod actuator based on an extended disturbance observer, Mechatronics 27: 47-56.

https://doi.org/10.1016/j.mechatronics.2015.02.003

4. Yao, B., Bu; F., Reedy, J.; Chiu, G. 2000. Adaptive robust motion control of single-rod hydraulic actuators: theory and experiments, IEEE/ASME Transactions on Mechatronics 5(1): 79-91. http://dx.doi.org/10.1109/3516.828592.

5. Shi, Z.; Gu, F.; Lennox, B.; Ball, A. 2005. The development of an adaptive threshold for model-based fault detection of a nonlinear electro-hydraulic system. Control Engineering Practice 13(11): 1357-1367. https://doi.org/10.1016/j.conengprac.2004.11.014.

6. Utkin, V.; Guldner, J.; Shi, J. 2009. Sliding Mode Control in Electro-Mechanical Systems: Taylor \& Francis Group.

7. Han, S.; Jiao, Z.; Wang, C.; Shang, Y. 2015. Fuzzy robust nonlinear control approach for electro-hydraulic flight motion simulator, Chinese Journal of Aeronautics 28(1): 294-304.

https://doi.org/10.1016/j.cja.2014.12.025.

8. Rezayi, S.; Arbabtafti, M. 2017. A new model-based control structure for position tracking in an electro-hydraulic servo system with acceleration constraint, Journal of Dynamic Systems, Measurement and Control 139(12): 12006-1-11.

http://dx.doi.org/ 10.1115/1.4036878.

9. Sirouspour, M.; Salcudean, S. 2001. Nonlinear control of hydraulic robots, IEEE Transactions on Robotics and Automation 17(2): 173-182. http://dx.doi.org/10.1109/70.928562.
10. Tang, R.; Zhang, Q. 2011. Dynamic sliding mode control scheme for electro-hydraulic position servo system, Procedia Engineering 24: 28-32.

https://doi.org/10.1016/j.proeng.2011.11.2596.

11. Chiang, M.; Chen, C.; Kuo, C. 2009. The high response and high efficiency velocity control of a hydraulic injection molding machine using a variable rotational speed electro-hydraulic pump-controlled system, International Journal of Advanced Manufacturing Technology 43(10): 841-851. http://dx.doi.org/10.1007/s00170-008-1759-z.

12. Alarçin, F. 2014. Nonlinear modelling of a fishing boat and fuzzy logic control design for electro-hydraulic fin stabilizer system, Nonlinear Dynamics 76(1): 581-590. http://dx.doi.org/ 10.1007/s11071-013-1152-9.

13. Cerman, O.; Hušek, P. 2012. Adaptive fuzzy sliding mode control for electro-hydraulic servo mechanism, Expert Systems with Applications 39(11): 10269-10277. http://dx.doi.org/10.1016/j.eswa.2012.02.172.

14. Yanada, H.; Furuta, K. 2007. Adaptive control of an electrohydraulic servo system utilizing online estimate of its natural frequency, Mechatronics 17(6):337-343. http://doi.org/10.1016/j.mechatronics.2007.04.007.

15. Mohanty, A.; Yao, B. 2011. Indirect adaptive robust control of hydraulic manipulators with accurate parameter estimates, IEEE Transactions on Control Systems Technology 19(3): 567-575. http://dx.doi.org/ 10.1109/TCST.2010.2048569.

16. Guo, K.; Wei, J.; Tian, Q. 2015. Nonlinear adaptive position tracking of an electro-hydraulic actuator, Proc IMechE Part C, Journal of Mechanical Engineering Science 229(17): 3252-3265. https://doi.org/10.1177/0954406214568821.

17. Huanqing, W.; Xiaoping, L.; Kefu, L. 2015. Adaptive neural data-based compensation control of non-linear systems with dynamic uncertainties and input saturation, IET Control Theory \& Applications 9(7):1058-1065. http://dx.doi.org/10.1049/iet-cta.2014.0709.

18. Liem, D.; Truong, D.; Park, H.; Ann K. 2016. A feedforward neural network fuzzy grey predictor-based controller for force control of an electro-hydraulic actuator, International Journal of Precision Engineering and Manufacturing 17(3): 309-321. https://doi.org/10.1007/s12541-016-0039-3.

19. Guo, Q.; Sun, P.; Yin J. 2016. Parametric adaptive estimation and backstepping control of electro-hydraulic actuator with decayed memory filter, Isa Transactions 62: 202-214. https://doi.org/10.1016/j.isatra.2016.02.009,

20. Xu, Z.; Ma, D.; Yao, J.; Ullah, N. 2016. Feedback nonlinear robust control for hydraulic system with disturbance compensation, Proceedings of the Institution of Mechanical Engineers, Part I: Journal of Systems and Control Engineering 230(9): 978-987. https://doi.org/10.1177/0959651816661450.

21. Guo, Q.; Shi, G.; Wang, D.; He, C. 2017. Neural network-based adaptive composite dynamic surface control for electro-hydraulic system with very low velocity, Proc IMechE Part I: Journal of Systems and Control Engineering 231(10): 867-880. https://doi.org/10.1177/0959651817731976. 
Mingxing YANG, Qi ZHANG, Xinliang Lu, Ruru XI, Xingsong WANG

\section{ADAPTIVE SLDING MODE CONTIONAL OF A NON- LINEAR ELECTRO-HYDRAULIC SERVO SYSTEM FOR POSITION TRACING}

\section{S u m mary}

In view of the electro-hydraulic position servo system with parameter uncertainty and bounded disturbances, an improved adaptive sliding mode control scheme is proposed. The mathematical model of the valve-controlled system is first constructed with consideration of the external disturbance, matched and mismatched unknown parameters. Then, the parametric adaptive estimation laws are established by Lyapunov technique to estimate the generalized uncertainty parameters, and the discontinuous projection algorithm is used to ensure the boundedness of parameter estimation. In order to eliminate the chattering phenomenon in sliding mode control, the saturation function is designed to replace the sign function and the gain coefficient is adjustable on the sliding surface. Finally, the comparative experimental results clarify that the proposed control scheme has better control performance than the PID controller and the SMC controller.

Key words: Electro-hydraulic servo system, parameter uncertainty, bounded disturbances, adaptive sliding mode control, trajectory tracing.

Received February 25, 2019

Accepted August 26, 2019 